\title{
Metodología Algorítmica para el Diseño Inherentemente Seguro de Planta
}

\section{Algorithmic Methodology for Inherently Safe Plant Design}

\section{Santiago Orellano}

Centro de Aplicaciones Informáticas y Modelado en Ingeniería (CAIMI), Facultad Regional Rosario, Universidad Tecnológica Nacional - Argentina.

sorellano@frro.utn.edu.ar

\section{Néstor Hugo Rodríguez}

Centro de Aplicaciones Informáticas y Modelado en Ingeniería (CAIMI), Facultad Regional Rosario, Universidad Tecnológica Naciona - Argentina.

nestorhugo_r@yahoo.com

\section{Nicolás José Scenna}

Centro de Aplicaciones Informáticas y Modelado en Ingeniería (CAIMI), Facultad Regional Rosario, Universidad Tecnológica Nacional - Consejo Nacional de Investigaciones Científicas y Técnicas (CONICET) - Argentina.

nscenna@yahoo.com.ar

\section{Resumen}

Los principios del diseño inherentemente seguro imponen la consideración del riesgo desde etapas tempranas. Generalmente se lo considera en las últimas etapas, independientemente de la optimización de layout, aun cuando existe una estrecha relación entre ambas. En este trabajo se aborda dicha problemática, proponiéndose modelos de programación mixta entera lineal (MILP) con el objetivo de optimizar la distribución del layout general de planta y una posible estrategia para vincular la síntesis de la playa de almacenamiento de sustancias combustibles. Hecho no reportado aún en la bibliografía especializada.

Se utiliza una estrategia jerárquica y secuencial para la contemplación de un elevado número de unidades de proceso considerando las limitaciones actuales de los métodos determinísticos para tal fin.

Se aplica el procedimiento a un caso de estudio particular, la optimización del layout de una planta de biodiesel incluyendo la optimización rigurosa de la playa de tanques de metanol. 
Palabras Claves: Layout, Almacenamiento de Combustibles, Diseño Inherentemente Seguro, MILP.

\begin{abstract}
Inherently safe design principles impose risk assessment from early stages of design. The consideration of risk is commonly made in the last design stage and is carried out independently of the layout optimization, even though there is a close relationship between both. In this work, this problem is addressed proposing models (Mixed Integer Linear Programming, 'MILP') to optimize the general plant layout and to introduce a possible strategy to the synthesis of combustibles tank farm. It isn't reported in the specialized bibliography yet.

A hierarchical and sequential strategy is used for the consideration of a large number of process units considering the current limitations of deterministic methods for this purpose.

The procedure was applied to a particular case of study, the optimization of a biodiesel plant layout, including the rigorous optimization of the methanol tank farm.
\end{abstract}

Keywords: Layout, Combustible Tank Farm, Inherently Safe Design, MILP.

\title{
Introducción
}

En las últimas décadas se publicaron diversos artículos que intentaron resolver el problema del layout a través de programación matemática y de la aplicación de técnicas de optimización. Surgieron, dos corrientes bien diferenciadas. Por un lado, se utilizaron métodos determinísticos de optimización (con la limitación de la cantidad de unidades posibles de tratar) (Jung et al., 2011; Medina-Herrera et al, 2014; entre otros) y por otro, se aplicaron técnicas de optimización meta-heurísticas (Genetics algorithm, Bat Methaheuristics algorithm, Tabu Search, Simulated annealing, entre otras) (Caputo, 2015; Latifi et al.,2017; entre otros) con la limitación de no poder garantizar los atributos de la solución óptima obtenida. La limitación de los métodos determinísticos motivó el desarrollo de técnicas heurísticas, como la metodología desarrollada por (Xu y Papageorgiou, 2007 y 2009), (Park et al., 2018), entre otras.

Es importante remarcar que, en las últimas décadas, se ha impuesto la sostenibilidad de los procesos; lo cual, entre otros factores, implica seguridad operativa y de diseño de los mismos. Entre los enfoques más importantes y consolidados en las últimas dos décadas podemos citar las estrategias utilizadas para la incorporación de la filosofía de diseño inherentemente seguro o bien de diseño basado en riesgo. La incorporación usual de la vulnerabilidad en el diseño del layout consiste en dotar de separaciones adecuadas a las unidades de procesamiento, cuantificando el impacto provocado por un evento accidental.

Los principios del diseño inherentemente seguro ponen especial énfasis en la minimización de los inventarios de sustancias con cierto riesgo asociado. Casal (2008) realiza un análisis histórico de los accidentes, determinando que el $17 \%$ de los mayores accidentes en la industria de proceso ocurrieron o involucraron a las instalaciones de almacenamiento. La NFPA (Nacional Fire and Protection Association de los Estados Unidos) reportó que en 2009 el $13 \%$ de los mayores incendios accidentales ocurrieron en tanques de almacenamiento. Estos números reflejan el riesgo asociado a estos sectores, y por lo tanto la necesidad de un tratamiento adecuado. Sin embargo, existen muy pocos trabajos que intenten vincular esta problemática con la obtención de modelos de optimización de layout, cuando en realidad 
están estrechamente relacionados. En general, la vinculación entre etapas tempranas del diseño de procesos y la optimización de layout es un campo de investigación poco explorado y muy prometedor (síntesis de procesos, de playas de almacenamiento, de layout de planta).

La problemática real que se enfrenta en la etapa del diseño del layout es, por un lado, la limitación de los métodos tradicionales para la resolución eficaz del problema, y por otro, la inclusión en el modelo de optimización de funcionales simples para el cálculo de las consecuencias asociadas a los eventos accidentales. En el caso de contarse con tales funciones, sería factible la toma de decisiones acoplándose dos etapas de diseño completamente desvinculadas (según estado del arte). Es decir, podrían vincularse fácilmente los niveles de impacto dadas las distancias (o viceversa) en función de las variables de diseño; siendo por lo tanto posible incluirlas en modelos de optimización de layout. Esto permitiría la toma de decisiones mencionadas tomando en cuenta un espacio amplio de variables de diseño y sus correspondientes niveles de vulnerabilidad del entorno.

\section{Desarrollo}

En general, la complejidad de este tipo de problemas responde a varios factores, entre los cuales pueden citarse el crecimiento combinatorio exponencial en función de las unidades de procesamiento (UP) a contemplar, la forma de obtener las distancias de separación (fijas o en función de modelos incorporados al problema), si se contemplan solo los interiores de la planta o se tienen en cuenta puntos vulnerables en el exterior, entre otros factores.

Con el objeto de simplificar el problema de la cantidad de UP a manejar (en los trabajos reportados en la bibliografía, no se involucran más de diez utilizando algoritmos determinísticos) incluimos una reformulación de la metodología propuesta por Xu y Papageorgiou (2007) que consiste en optimizar secuencialmente la ubicación de las UP en el terreno que ocupa la planta. Para ello se descompone el problema tomando la optimización primeramente de un subconjunto de n unidades. Una vez realizada, se fijan estas UP en las posiciones "óptimas resultantes" y se optimiza el grupo restante añadiendo etapa por etapa un grupo de m unidades secuencialmente, hasta ubicar la totalidad de las UP. En cada etapa de optimización, se fijan las posiciones de todas las unidades optimizadas previamente. Obviamente, la solución óptima del método descrito es sensible a los valores de $m$ y $n$ adoptados, los cuales están limitados por la capacidad de cómputo y, además, a las unidades escogidas en cada etapa del algoritmo; pero permite resolver adecuadamente problemas reales.

Por otra parte, existen unidades (como las unidades de almacenamiento de sustancias peligrosas) que son críticas en función del riesgo potencial subyacente. En este trabajo, para considerar el riesgo se define una etapa de pre-procesamiento en la cual se evalúan las unidades (UP) que conforman el proceso. Esto consiste en asignar un valor que se relaciona con su peligrosidad (dada una métrica específica) a cada uno de los distintos bloques/unidades UP. De tal listado, se toman las UP más peligrosas como problemas independientes de optimización. En general, los sistemas de almacenamiento de sustancias peligrosas son candidatos para esta selección, por lo que aquí se plantea la necesidad de la optimización del layout de las playas de tanques de almacenamiento de materias primas, productos intermedios y productos finales. Uno de los principales problemas al proponer una descomposición de un problema de optimización en distintas etapas jerárquicas es la obtención de funciones objetivo homogéneas a los efectos de obtener una secuencia de soluciones óptimas coherentes en cada etapa a resolver. Sin embargo, en la literatura se asumen volúmenes, posiciones 
y configuraciones establecidas para estos sistemas.

A continuación, se desarrolla el algoritmo propuesto y la aplicación a un caso de estudio particular; el diseño del layout general de una planta de biodiesel.

\section{Caso de Estudio: Layout de Planta de Biodiesel}

La planta tiene especificada como objetivo de diseño una producción de 1000 Ton/día. Dada la estructura del proceso y los parámetros de operación de la planta, es posible simular la misma, y obtener mediante resolución de los balances de materia y energía los consumos de materias primas y producción de subproductos y, a partir de esto, de acuerdo a los intervalos de tiempo de carga y descarga que se planteen pueden determinarse los volúmenes a almacenar en el sistema.

Las unidades consideradas se incluyen con sus respectivas dimensiones en la Tabla 1. La última columna establece la etapa en que se incluye según la metodología secuencial de resolución adoptada. Es necesario aclarar, además, que las dimensiones de la playa de tanques de metanol y su distribución es resultado del modelo de optimización que desarrollaremos a continuación.

\begin{tabular}{|l|c|c|c|c|}
\hline \multicolumn{1}{|c|}{ Unidad } & $\begin{array}{c}\text { Número } \\
\text { ID }\end{array}$ & I1 (m) & I2 (m) & Etapa \\
\hline Playa de tanques de metanol & $(1)$ & 27,5 & 27,5 & II \\
\hline Sala de calderas & $(2)$ & 28 & 38 & I \\
\hline Playa de tanques de glicerina & $(3)$ & 13 & 26 & $I$ \\
\hline Planta de producción & $(4)$ & 60 & 65 & $I$ \\
\hline Playa de tanques de Aceite & $(5)$ & 32 & 32 & I \\
\hline Playa de tanques de Biodiesel & $(6)$ & 32 & 32 & $I$ \\
\hline Planta de refinación & $(7)$ & 26 & 68 & $I$ \\
\hline Planta de pretratamiento & $(8)$ & 17 & 27 & $I$ \\
\hline Torres enfriamiento & $(9)$ & 28 & 38 & II \\
\hline Planta de glicerina & $(10)$ & 26 & 38 & II \\
\hline Pileta para tratamiento de efluentes & $(11)$ & 24 & 33 & II \\
\hline Laboratorio & $(12)$ & 10 & 20 & II \\
\hline Ingreso-Oficinas & $(13)$ & 20 & 20 & II \\
\hline Taller & $(14)$ & 20 & 20 & II \\
\hline Sala control & $(15)$ & 10 & 10 & II \\
\hline
\end{tabular}

Tabla 1. Unidades de la Planta de Biodiesel

En primer lugar, se realiza en la etapa de pre-procesamiento, la evaluación de la peligrosidad de las UP, siendo en este caso la más importante la unidad de almacenamiento de metanol, crítica en función del riesgo potencial subyacente. 


\section{Primera Etapa. Optimización del layout y distribución de la playa de almacenamiento de metanol}

Para la optimización de la síntesis y layout se considera, además de los costos directamente vinculados con la misma, el costo del área exterior potencialmente afectada debido a la ocurrencia de un accidente (en este caso particular el área sujeta a una radiación superior a $15 \mathrm{Kw} / \mathrm{m}^{2}$ frente a la ocurrencia de un trench fire -incendio de trinchera-). Este flujo calorífico se establece considerando el límite propuesto por Landucci y Cozzani, (2009) para evitar la ocurrencia de efecto dominó. Se compatibilizan de esta manera las funciones objetivo utilizadas en ambas etapas secuenciales de resolución. Es decir, se determina el layout y la decisión de la construcción de paredes internas; considerando no sólo los costos de construcción sino también el área externa a la playa que sería afectada ante un eventual incendio de modo de garantizar la no instalación de unidades en la posterior optimización del layout general en dicha área. Este problema (y su solución) no han sido aún reportados en la literatura.

En este problema sólo se optimiza una función económica (costo) considerando la vulnerabilidad del entorno (radiación límite); a través de la implementación en un modelo MILP, el cual es resuelto en GAMS. Esto implica obtener un área externa segura al contorno de la playa dada la restricción de radiación máxima impuesta. Este modelo contiene 1224 variables (920 binarias, 71 discretas y 233 continuas); 9881 restricciones (721 igualdades y 9160 desigualdades) y es resuelto con el solver SCIP en 3 minutos, 15 segundos. El costo de la playa de tanques tiene en cuenta el terreno, el endicamiento (si existe) y los tanques resultantes. Si existen (en la solución) tanques embebidos en la llama, se considera, además, el costo de reposición de los mismos (ponderado por su probabilidad de ocurrencia) -para mayor detalle ver (Orellano et al., 2019a)-. Estas variables, a nuestro conocimiento, no son tenidas en cuenta en los problemas de layout de planta completa reportados en la literatura.

El campo de radiaciones en el exterior de la playa de tanques (dada una potencial fuga y posterior incendio del combustible derramado), ha sido calculado a través de un modelo previamente desarrollado por el grupo de trabajo (CAIMI), asegurando estimaciones conservadoras para la determinación del campo de iso-radiación a un nivel especificado. En el modelo de optimización, este campo ha sido aproximado a través de un octágono irregular con el objetivo de considerar el efecto de borde característico del incendio de charcos contenidos/endicados (trench fire) sin perder la linealidad del modelo de optimización (Fig. 1). En la literatura se asume geometría "cilíndrica" del charco, no existente en las plantas reales.

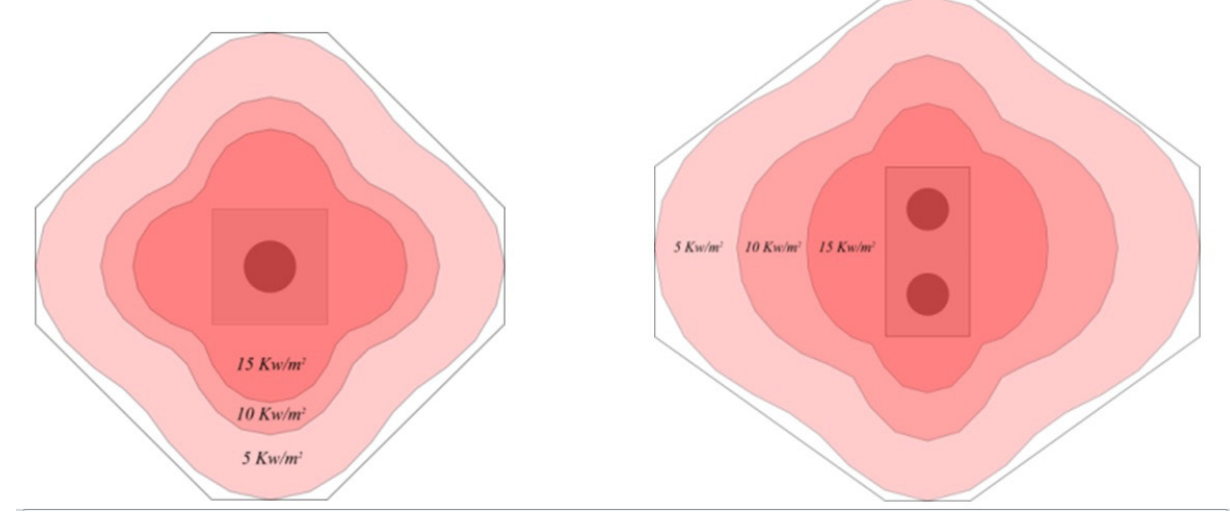

Fig. 1: Campos de iso-radiación 
La legislación aplicable al almacenamiento de sustancias potencialmente peligrosas establece restricciones que limitan la distancia entre tanques y el volumen del endicamiento que los contiene. La ley 13.660 aplicable a playas de almacenamiento de combustibles, establece en los artículos 321 y 329 estas restricciones, que fueron incluidas como restricciones en el modelo de optimización.

Dado un volumen de sustancia a almacenar, determinado como mencionamos anteriormente, la estructura de la playa de tanques óptima se deriva de la "superestructura" mostrada en la Fig. 2. Es decir, se diseñan los mismos para contener el volumen total (si se utiliza un tanque, el mismo tendrá 4 veces el volumen de cada tanque, en caso de que se hubiesen utilizado cuatro). Debe remarcarse que el impacto externo se reduce al dividir el inventario que provoca el incendio, pero aumenta el costo de la instalación de almacenamiento. La determinación de la superestructura se deriva del análisis de las soluciones óptimas. Dado el volumen a almacenar, el número máximo de tanques a incorporar en la superestructura (Fig. 3) es fácilmente deducible; ya que el costo total óptimo no contempla un número muy grande de los mismos, según reglas heurísticas, y de las soluciones de varios ejemplos resueltos. En general con la configuración "extrema" indicada para el volumen total a almacenar se obtiene rápidamente la solución óptima.

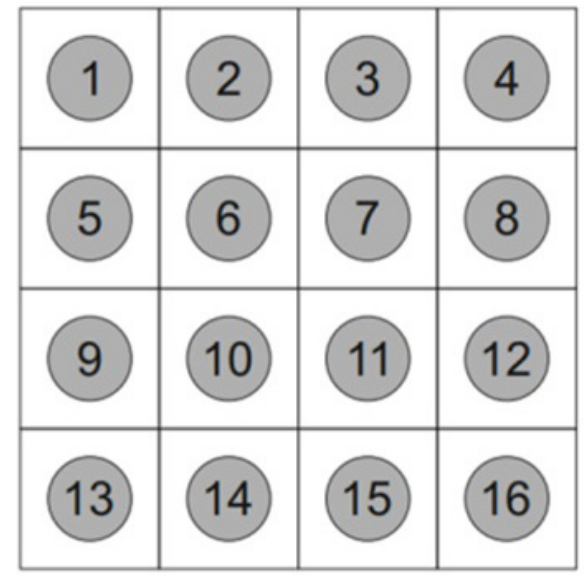

Fig. 2: Esquema base

La presencia de endicamientos se ha modelado a través de un grafo en el que los nodos del mismo representan a los posibles tanques, y los arcos a las paredes del endicamiento presentes entre ambos -para más detalle del modelo ver (Orellano et al., 2019a)-.

Se ha adoptado el valor de $\mathrm{P}=10^{-4}$ (Tanques. año) ${ }^{-1}$ (HSE-UK, 2012), para la tasa de fallas para derrames en tanques con contenido inflamable. Considerando la probabilidad de falla de un tanque independiente de la del resto, entonces la probabilidad que uno de un conjunto de tanques falle (fuga), es fácilmente calculable. A partir de esto, es posible cuantificar en términos económicos la destrucción provocada, ponderada por la probabilidad de ocurrencia, y su consecuente inclusión en la función objetivo.

En cuanto a la "vulnerabilidad del espacio exterior" de la playa, se utilizó el modelo riguroso (no puntual, ya que es muy simplificado) de incendio de charco previamente mencionado para calcular el área externa sujeta a una radiación superior o igual a la fijada para 
evitar efecto dominó $\left(15 \mathrm{Kw} / \mathrm{m}^{2}, t \geq 10 \mathrm{~min}\right)$.

La función objetivo incluye el costo (anualizado) de los tanques; del área ocupada (terreno y construcción de la base del endicamiento); costo de construcción de las paredes del endicamiento, de cañerías, el asociado a la vulnerabilidad interna de la playa y el área externa a la playa sujeta a un nivel de radiación superior a $15 \mathrm{Kw} / \mathrm{m}^{2}$. El problema se resuelve paramétricamente en cuanto al número de tanques y la cantidad que se disponen por endicamiento. El modelo implementado brinda como resultado las distintas disposiciones óptimas (número de tanques y modo en que se agrupan), indicadas a continuación. En la Fig. 3 se observa el costo óptimo de la disposición de la playa de almacenamiento para distinto número de tanques considerados. Se observa la presencia de un óptimo mediante la utilización de 4 tanques. La distribución óptima asociada se muestra en la Fig. 4, observándose la existencia de las paredes intermedias divisoras en el dique (en la solución óptima).

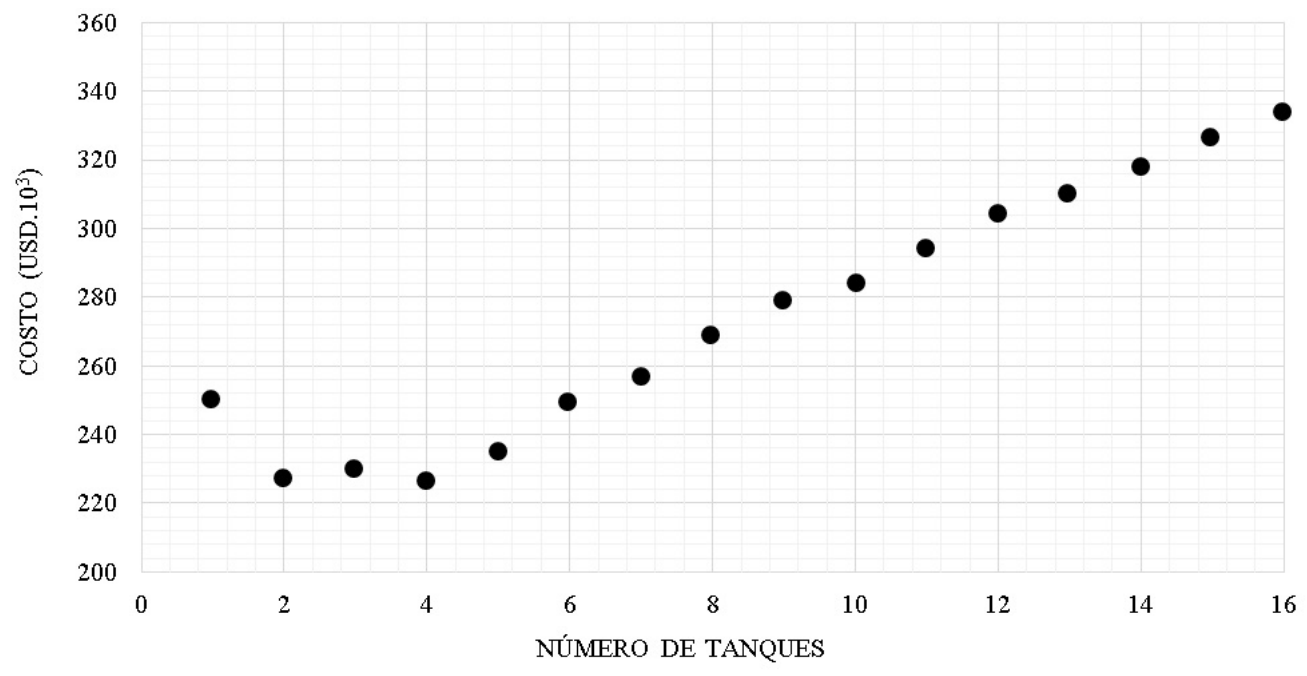

Fig. 3: Costos óptimos de la playa de tanques de metanol

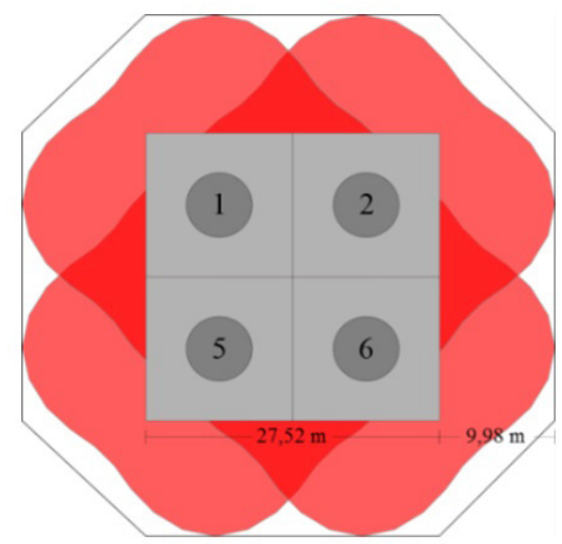

Fig. 4: Disposición óptima de la playa 


\section{Segunda Etapa. Algoritmo para la optimización del layout de planta}

Dados los datos disponibles en este caso de estudio (Tabla 1), y los datos óptimos obtenidos para la playa de almacenamiento en la primera etapa de la optimización, se procede a continuación a resolver el modelo de optimización del layout global.

Se enumeran resumidamente (dadas las limitaciones de espacio) las principales consideraciones realizadas en el modelo de optimización:

a) Forma: Se utiliza la simplificación de considerar las unidades de planta como los rectángulos en los que están inscriptas, de dimensiones $a_{i} \cdot b_{i}$.

b) Rotación: A través de la variable binaria $\left(\mathrm{p}_{\mathrm{i}}\right)$, se consideran dos posibles orientaciones para cada unidad o instalación, ambas con sus lados paralelos a los ejes de coordenadas.

c) Distancias mínimas entre unidades: Se incorpora la decisión conservadora asumiendo que la distancia mínima establecida $\left(d_{i, j}^{\min }\right)$ se cumpla en al menos una de las dos direcciones coordenadas. Esta relajación permite que el modelo no pierda la linealidad, lo que ocurriría al utilizar distancias euclideas.

Las distancias mínimas entre las secciones de planta, a excepción de la playa de tanques de metanol, fueron adoptadas de acuerdo a los distanciamientos mínimos establecidos por la secretaría de energía en la resolución 1296/2008. La playa de tanques de metanol fue diseñada de acuerdo al modelo de optimización mencionado y la distancia de impacto es función de este diseño adoptado.

En cuanto a las distancias consideradas desde la playa de almacenamiento de metanol, además de la calculada por el trench fire, otro escenario accidental probable se corresponde con el derrame del líquido y la evaporación del mismo formando una nube tóxica. Se calcula la distancia a la cual se manifestaría la concentración correspondiente al ERPG-3 (-Emergency Response Planning Guidelines-, que es la máxima concentración en aire por debajo de la cual se cree que casi todos los individuos pueden estar expuestos hasta una hora sin experimentar o desarrollar efectos que amenacen su vida) debido a la difusión de metanol en las condiciones ambientales más adversas (elevada estabilidad atmosférica y una velocidad de "viento calmo"). Se dispone, por lo tanto, de las distancias mínimas a verificarse entre la playa óptima de tanques de metanol y las demás áreas, ya sea las de frecuente presencia de personas (oficinas, talleres, sala de control, laboratorios), como las de operaciones, a ser incorporadas en el layout total de planta. Las distancias a verificarse desde esta playa, que es la que mayores distancias de impacto tiene asociada, se han aproximado con mayor rigurosidad a través de un octágono.

d) Distancia a los límites de la planta: Se consideran las distancias mínimas requeridas entre los bordes de las instalaciones y los límites del terreno, tal como exige la legislación aplicable a los distintos tipos de plantas. En el caso del metanol, si se considera la vulnerabilidad del entorno a la planta es razonable confinar el nivel de ERPG-3 para la dispersión de tóxicos a los límites de la planta (Orellano y Scenna, 2019b).

En la estrategia de descomposición, el problema de la adopción del primer conjunto de UP a optimizar es crítico. Se propone, para la determinación de las unidades correspondientes al primer grupo de optimización, un modelo en GAMS, a través del cual se determina la combinación de UPs, de modo que agrupadas en conjuntos de $n$ unidades, se maximice el costo unitario de la conectividad entre ellas. En general, las unidades de proceso, debido a la elevada conectividad hacia las demás unidades de la planta (playas de almacenamiento, plantas de subproductos, plantas de tratamiento de efluentes, entre otras) asumen una posición central en el layout (para más detalle ver -Orellano y Scenna, 2019º-; -Xu y Papageorgiou 
,2007-). A partir de esto es razonable que las unidades de proceso estén incluidas en el primer grupo de optimización ( $n$ ), (tal como se verifica en los resultados obtenidos).

Sea, Costo $_{i, j}^{\text {conect }}$ el costo de la unidad de conexión entre las unidades i y j. Se define la variable binaria para determinar si la unidad i forma parte del primer grupo de $n$ unidades (UP) de optimización. Luego, se modela mediante la relajación BigM (Ec. (1) a (4)):

$$
\begin{array}{ll}
\operatorname{máx.} & Z=\sum_{i} \sum_{j} C_{i, j} \\
\text { s.t. } & y_{i} \wedge y_{j} \Rightarrow C_{i, j}=\text { Costo }_{i, j}^{\text {conect }} \\
& \neg\left(y_{i} \wedge y_{j}\right) \Rightarrow C_{i, j}=0 \\
& \sum_{i} y_{i}=n
\end{array}
$$

Donde, la variable $\mathrm{C}_{\mathrm{i}, \mathrm{j}}$ representa el costo de conexión unitario entre las unidades i y j en el caso de que las mismas sean incluidas en el primer bloque de optimización.

Una vez determinado el conjunto de unidades que se incluirán en el primer bloque de optimización, se fija la posición de una de las unidades del primer bloque en el centro de coordenadas. Lo que permite que el layout se expanda en todas las direcciones, a diferencia de las formulaciones tradicionales del problema (en uno de los vértices). Se incluyeron en la primera etapa del algoritmo las unidades (2) a (8), que fueron los resultados del modelo de optimización utilizado para el pre-procesamiento y se fijaron las coordenadas de la planta de producción en el centro de coordenadas como mencionamos. El modelo utilizado para la optimización de la distribución de estas $n$ unidades se describe brevemente mediante las Ec. (5) a (28). Este se basa en una ampliación y adecuación del modelo desarrollado por (Tsai et al., 1993).

$$
\begin{array}{lc}
{\left[\begin{array}{c}
R_{i, j} \\
x_{i} \geq x_{j}+s p_{i, j}^{x}+d_{i, j}^{\min }
\end{array}\right] \vee\left[\begin{array}{c}
L_{i, j} \\
x_{j} \geq x_{i}+s p_{i, j}^{x}+d_{i, j}^{\text {min }}
\end{array}\right] \vee\left[\begin{array}{c}
U_{i, j} \\
y_{i} \geq y_{j}+s p_{i, j}^{y}+d_{i, j}^{\min }
\end{array}\right] \vee\left[\begin{array}{c}
D_{i, j} \\
y_{j} \geq y_{i}+s p_{i, j}^{y}+d_{i, j}^{\text {min }}
\end{array}\right] i<j} \\
s p_{i, j}^{x}=0,5\left[a_{i} p_{i}+b_{i}\left(1-p_{i}\right)+a_{j} p_{j}+b_{j}\left(1-p_{j}\right)\right] & i<j \\
s p_{i, j}^{y}=0,5\left[b_{i} p_{i}+a_{i}\left(1-p_{i}\right)+b_{j} p_{j}+a_{j}\left(1-p_{j}\right)\right] & i<j
\end{array}
$$

Las disyunciones mostradas (5) establecen como necesidad que la distancia mínima a satisfacerse entre unidades $\left(d_{i, j}^{\min }\right)$ se cumpla en al menos una de las direcciones coordenadas; esto es, $\mathrm{R}_{\mathrm{i}, \mathrm{j}}$ si la distancia mínima se cumple en la dirección horizontal con la unidad $i$ a la derecha de la unidad $j, L$ a la izquierda, $U$ arriba y $D$ debajo. Donde $s p_{i, j}^{x}$ representa la suma de la mitad de los lados en esa dirección; esta es variable porque se permite rotación, considerada a través de la variable binaria $p$. Mediante la relajación BigM, estas disyunciones pueden expresarse mediante las Ec. (8) a (12): 


$$
\begin{array}{lc}
x_{i} \geq x_{j}+s p_{i, j}^{x}+d_{i, j}^{\min }-M\left(1-r_{i, j}\right) & i<j \\
x_{j} \geq x_{i}+s p_{i, j}^{x}+d_{i, j}^{\min }-M\left(1-l_{i, j}\right) & i<j \\
y_{i} \geq y_{j}+s p_{i, j}^{y}+d_{i, j}^{\min }-M\left(1-u_{i, j}\right) & i<j \\
y_{j} \geq y_{i}+s p_{i, j}^{y}+d_{i, j}^{\min }-M\left(1-d_{i, j}\right) & i<j \\
r_{i, j}+l_{i, j}+u_{i, j}+d_{i, j} \geq 1 & i<j
\end{array}
$$

Donde $r, l, u$ y $d$ representan las variables binarias asociadas a cada disyunción.

Es necesario considerar, para incluir el costo de la conectividad, la distancia entre las unidades. La distancia utilizada para tal fin fue la distancia Manhattan, por dos razones. Por un lado, por la forma de disponer las tuberías en planta y por otro, porque permite mantener la linealidad del modelo. Incluir la determinación de esta distancia, requiere el cálculo de $\left|x_{i}-x_{j}\right|+\left|y_{i}-y_{j}\right|$. Para evitar el valor absoluto y la programación disyuntiva, se requiere de la definición de las variables binarias $r_{i, j}^{\prime}, l_{i, j}^{\prime}, u_{i, j}^{\prime}, d_{i, j}^{\prime}$ para establecer la posición relativa entre las unidades i y $\mathrm{j}$.

$$
\begin{array}{lc}
{\left[\begin{array}{c}
R_{i, j}^{\prime} \\
x_{i} \geq x_{j}
\end{array}\right] \vee\left[\begin{array}{c}
L_{i, j}^{\prime} \\
x_{j} \geq x_{i}
\end{array}\right]} & i<j \\
{\left[\begin{array}{c}
U_{i, j}^{\prime} \\
y_{i} \geq y_{j}
\end{array}\right] \vee\left[\begin{array}{c}
D_{i, j}^{\prime} \\
y_{j} \geq y_{i}
\end{array}\right]} & i<j
\end{array}
$$

Las cuales se modelan, de manera similar a la disyunción anterior, mediante la relajación BigM. Definidas las variables binarias mencionadas, se calcula la distancia mediante las Ec. (15) a (19):

$$
\begin{array}{lc}
\operatorname{dist}_{i, j}=\operatorname{dist}_{i, j}^{x}+\operatorname{dist}_{i, j}^{y} & i<j \\
\operatorname{dist}_{i, j}^{x} \geq x_{i}-x_{j}-M\left(1-r_{i, j}^{\prime}\right) & i<j \\
\operatorname{dist}_{i, j}^{x} \leq x_{i}-x_{j}+M\left(1-r_{i, j}^{\prime}\right) & i<j \\
\operatorname{dist}_{i, j}^{x} \geq x_{j}-x_{i}-M\left(1-l_{i, j}^{\prime}\right) & i<j \\
\operatorname{dist}_{i, j}^{x} \leq x_{j}-x_{i}+M\left(1-l_{i, j}^{\prime}\right) & i<j
\end{array}
$$

De manera análoga se define la distancia en la dirección del eje y.

Para independizar el modelado de un vértice, como suele plantearse el problema típico de layout, se incluyen las siguientes definiciones, donde $\mathrm{X}_{t}$ e $\mathrm{Y}_{t}$ representan las longitudes de los lados del terreno: 


$$
\begin{array}{lc}
X_{t}^{+} \geq x_{i}+0,5 a_{i} p_{i}+0,5 b_{i}\left[1-p_{i}\right]+d_{i}^{\text {lim }} & i=1,2, \ldots, n \\
X_{t}^{-} \leq x_{i}-0,5 a_{i} p_{i}-0,5 b_{i}\left[1-p_{i}\right]-d_{i}^{\text {lim }} & i=1,2, \ldots, n \\
Y_{t}^{+} \geq y_{i}+0,5 a_{i}\left[1-p_{i}\right]+0,5 b_{i} p_{i}+d_{i}^{\text {lim }} & i=1,2, \ldots, n \\
Y_{t}^{-} \geq y_{i}-0,5 a_{i}\left[1-p_{i}\right]-0,5 b_{i} p_{i}-d_{i}^{\text {lim }} & i=1,2, \ldots, n \\
X_{t}=X_{t}^{+}-X_{t}^{-} & i=1,2, \ldots, n \\
Y_{t}=Y_{t}^{+}-Y_{t}^{-} & i=1,2, \ldots, n
\end{array}
$$

La función objetivo en esta etapa se corresponde con la Ec. (26):

$$
\text { Costo }=X_{t} Y_{t} c_{t}+C_{c o n}^{t}
$$

Donde, $c_{t}$ representa el costo unitario del terreno y $C_{c o n}^{t}$ costo total de conectividad, el cual se determina a través de Ec. (27) y (28).

$$
\begin{array}{ll}
C_{\text {con }}^{t}=\sum_{i=1}^{n} \sum_{j=1}^{n} C_{i, j}^{\text {conect }} \cdot l_{i, j}^{\text {conect }} & i \neq j \\
l_{i, j}^{\text {conect }}=\left[\operatorname{dist}_{i, j}^{x}+\operatorname{dist}_{i, j}^{y}\right] \operatorname{conex}(i, j) & i \neq j
\end{array}
$$

El producto de las longitudes de los lados del terreno genera la no linealidad y la no-convexidad de la función objetivo, lo que dificulta la optimización. Este producto bilineal ha sido linealizado a través de la relajación de McCormick. Para realizarlo se recurrió a la división del dominio original en subdominios, y se modeló la disyunción generada en cada iteración a través del convex hull (Balas, 1998; Vecchietti et al., 2003; Ruiz y Grossman, 2012).

Como se ha expresado, en las etapas de optimización del layout, se fijan las posiciones y orientaciones obtenidas en etapas anteriores y se incluye la optimización de un grupo $(m)$ de unidades adicionales. La resolución del caso de estudio es factible en tiempos razonables en 2 etapas, se incluyen a continuación los resultados obtenidos luego de realizar ambas etapas de optimización.

\section{Resultados}

Se muestra a continuación (Fig. 5) el tiempo de resolución y el valor de la función objetivo (costo) en función del número de unidades incluidas en la primera etapa de optimización. Para $n \geq 9$, la solución factible encontrada fue peor a la obtenida comenzando con 7 y 8 unidades. Las soluciones obtenidas con $n=7$ y $n=8$ presentan resultados similares, siendo éstas las soluciones más adecuadas halladas.

Es necesario resaltar que no se encontraron soluciones superadoras mediante la selección 
aleatoria de las unidades para la primera etapa de optimización.

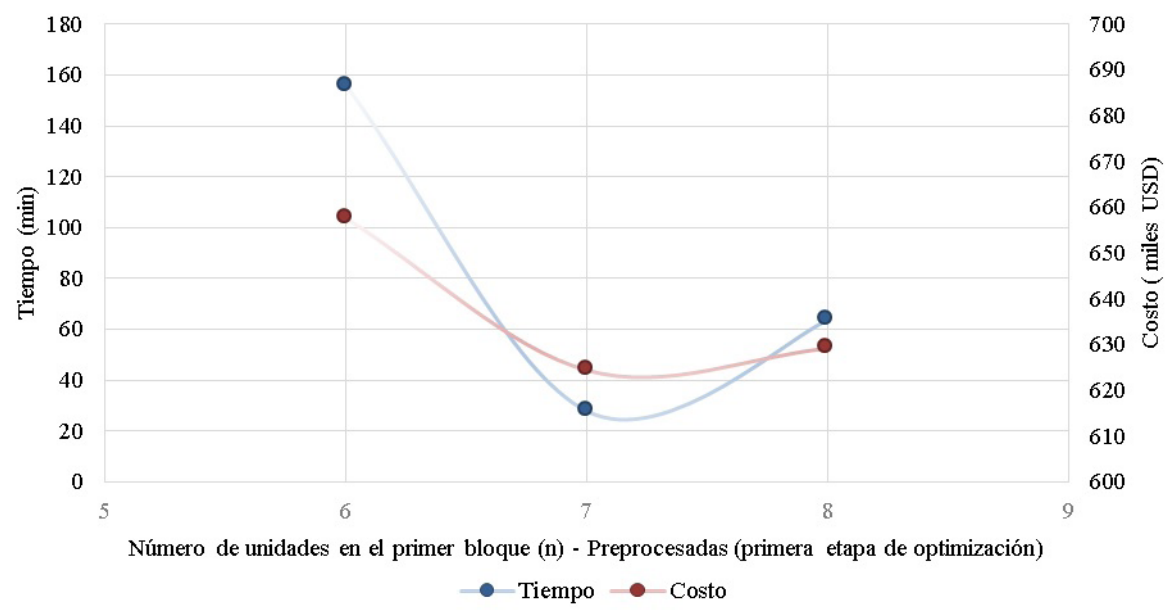

Fig. 5: Objetivo y tiempo de resolución en función de n.

Se incluyen a continuación los layouts obtenidos en ambas etapas de optimización (Fig. 6 y 7$)$.

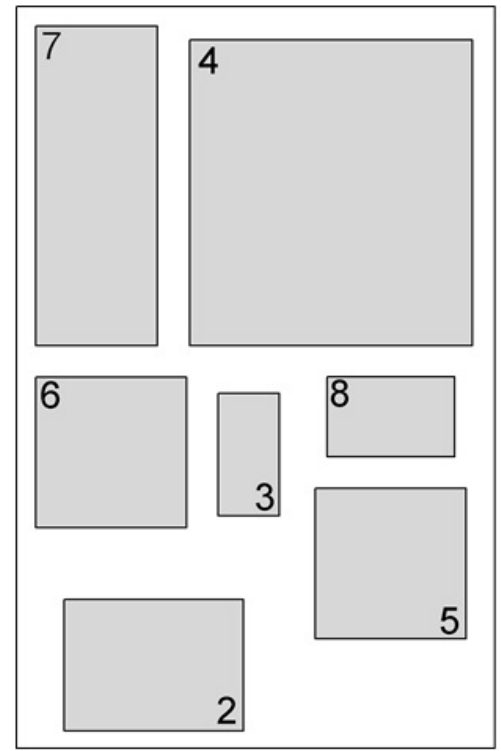

Fig. 6: Primera etapa del algoritmo de optimización de layout

Se observa una secuencia esperable de las UP a través de la cadena $5 \rightarrow 8 \rightarrow 4 \rightarrow 7 \rightarrow 6$, que correspondería respectivamente a la playa de tanques de aceite, la planta de pre-tratamiento, 
la planta de producción de Biodiesel, la planta de refinación y la playa de tanques de biodiesel. Se observa, además, el apartamiento de la sala de calderas, dada por las distancias reguladas por la secretaría de energía considerando el riesgo asociado a las mismas frente a una potencial explosión.

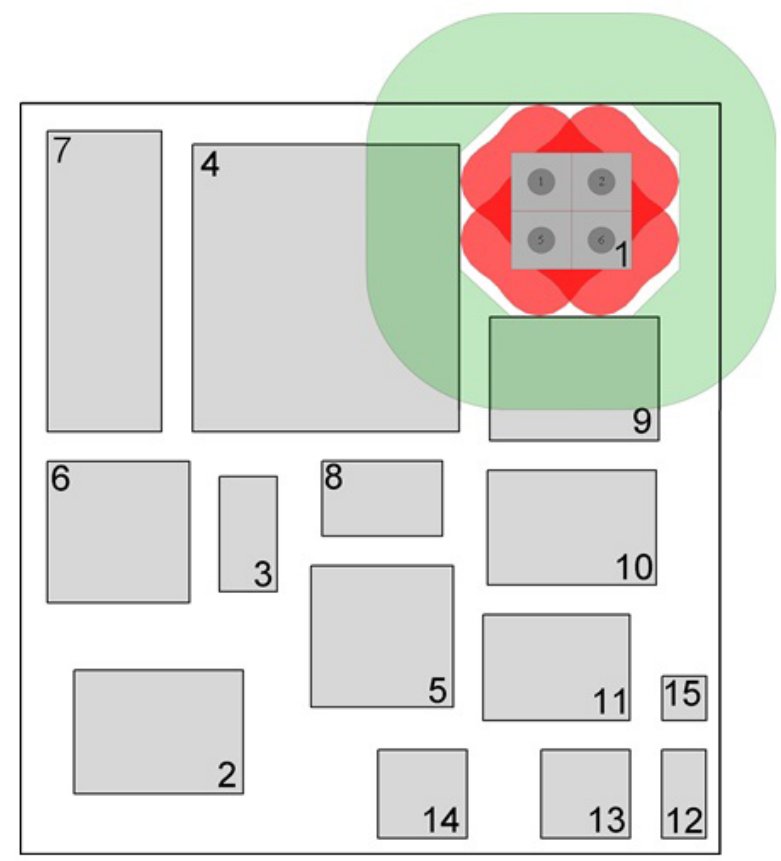

Fig. 7: Segunda etapa del algoritmo

Una vez concluida la segunda etapa del algoritmo (fijando las UP según la Fig. 6, e incorporando las restantes para la optimización de su ubicación), se obtiene el layout óptimo para la totalidad de las UP. El layout obtenido presentó dimensiones de $157 \mathrm{~m}$ x 169,5 m. En el óptimo, la relación entre los costos asociados a la interconexión y al costo del terreno con el costo total fueron: $36,07 \%$ (costo de la conectividad) y 63,93 \% (costo del terreno).

Analizando la distribución de las UP, se observa la ubicación en un extremo de la playa de tanques de metanol, debido a que en el modelo no se consideró la vulnerabilidad del entorno del complejo industrial. En caso de considerarse puntos de interés en el exterior del complejo industrial, la misma adoptaría posiciones centrales con el objetivo de confinar los niveles correspondientes al ERPG-3 dentro de los límites de la planta - esto se puede observar en (Orellano y Scenna, 2019) ). Obviamente será necesario, en caso de considerarse ésta, la redefinición de los criterios de pre-procesamiento adoptados, considerando para su determinación, no sólo las UP que presenten mayor costo de interconexión, sino también el riesgo asociado a las mismas. En la Fig. 8 se incluye a modo ilustrativo el esquema de interconectividad utilizado. 


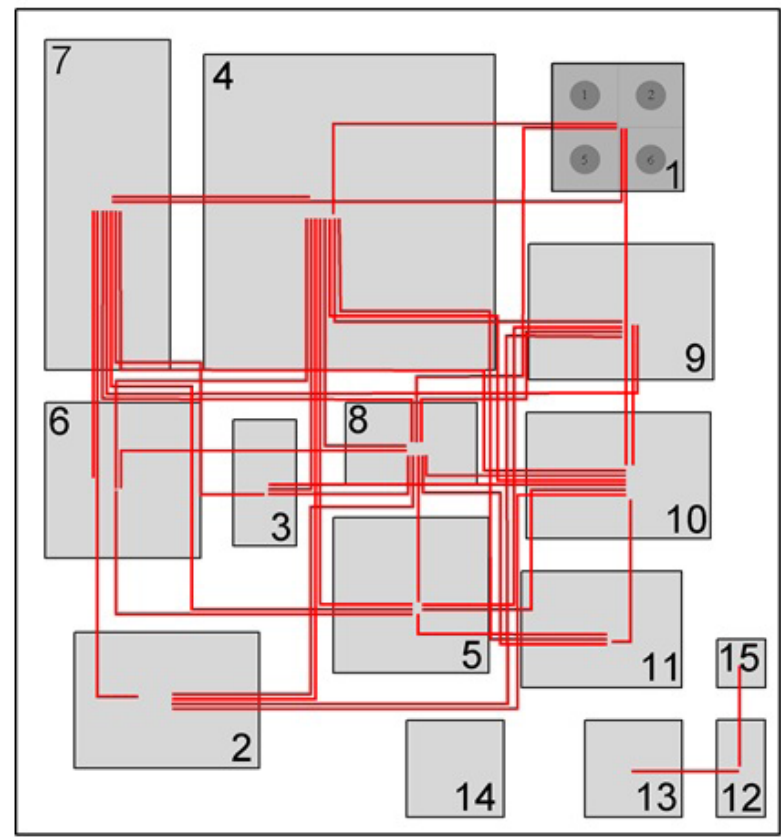

Fig. 8: Diagrama de conexiones

\section{Conclusiones}

Existen escasos trabajos y/o metodologías difundidas que permitan en lo posible acoplar la toma de ciertas decisiones en la etapa de síntesis y diseño del layout considerando el riesgo. Este trabajo, aún con ciertas limitaciones evidentes, por ejemplo, la optimización por etapas que podría llevar a la obtención de óptimos no globales, sienta las bases para desarrollar una metodología para la obtención del layout óptimo considerando el diseño basado en riesgos; permitiendo además tomar en cuenta simultáneamente, variables correspondientes a etapas tempranas de diseño (de la ingeniería conceptual). Es necesario destacar que no solo no existe reportada tal metodología, y las herramientas que permitan su ejecución; sino que el modelo utilizado para la optimización de la playa de almacenamiento de metanol, y su integración al problema del layout, no han sido aún exploradas según nuestro conocimiento.

Resulta importante entonces, proponer pasos en la dirección del diseño óptimo del layout basado en riesgos. Futuros trabajos deberían incluir correlaciones (o modelos reducidos) para la estimación de distancias de impacto para todos los potenciales eventos en industrias de proceso. Estos modelos, deberían ser lo suficientemente precisos, ya que de ello depende la bondad de la solución óptima obtenida; y a su vez lo suficientemente sencillos como para ser incorporados en un modelo de optimización, evitando la incorporación de excesivas no linealidades y pérdidas de convexidad.

Es evidente que las decisiones adoptadas en la etapa de síntesis del proceso, playa de tanques, entre otros, impactan directamente en el posterior diseño del layout. A través de este trabajo se discuten notorios avances tendientes a mitigar la desvinculación existente entre estas de diseño estrechamente relacionadas considerando la filosofía de diseño basado en riesgos. Sin embargo, si existieran playas que combinan varias sustancias el método debiera 
contemplar dicha cuestión.

En cuanto al algoritmo secuencial de incorporación de UP, se observa una eficiente respuesta mediante un pre-procesamiento de las unidades. Sin embargo, sería interesante en futuros trabajos comparar las soluciones obtenidas a través de esta técnica con las soluciones alcanzadas por algoritmos heurísticos para validar los resultados. En cuanto al criterio de inicialización, en futuros trabajos deberían desarrollarse lineamientos generales para la selección de las unidades iniciales y del orden de secuenciación en función de criterios no sólo económicos (conectividad) sino también considerando la potencial afectación de las UP al entorno de la planta y considerando la mitigación del efecto dominó (riesgo). 


\section{Referencias}

Balas, E. (1998). Disjunctive programming: Properties of the convex hull of feasible points. Discrete Applied Mathematics 89: 3-44.

Caputo, A.C., Pelagagge, P.M., Palumbo, M., Salini, P., (2015). Safety-based process plant layout using genetic algorithm. Journal of Loss Prevention in the Process Industries, 34: $139-150$.

Casal, J. (2008). Evaluation of the Effects and Consequences of Major Accidents in Industrial Plants. Elsevier, Amsterdam, Netherlands.

EPA (2016). ALOHA (5.4.7). https://www.epa.gov/cameo/aloha-software

Failure Rate and Event Data for use within Risk Assessments (2012) - HSE-UK

Jung, S. Facility sitting and layout optimization based on process safety. Thesis for the degree of Doctor of Philosophy, Texas A\&M University. (2010).

Landucci G., Cozzani V. (2009). The assessment of the damage probability of storage tanks in domino events triggered by fire. Accident Analysis and Prevention 41: 1206-1215.

Latifi, S., Mohammadi, E., Khakzad N. (2017). Process plant layout optimization with uncertainty and considering risk. Computers and Chemical Engineering, 106: 224-242

Ley $n^{\circ} 13.660$. Decreto $n^{\circ} 10.877 / 60$. Seguridad de las instalaciones de elaboración, transformación y almacenamiento de combustibles sólidos minerales, líquidos y gaseosos.

Medina-Herrera, N., Jiménez, G., Grossman, I.E. (2014). A mathematical programming model for optimal layout considering quantitative risk analysis. Computers and Chemical Engineering, 68: 165-181.

Orellano, S.; Scenna, N. J. (2018). Optimización de layout considerando el diseño inherentemente seguro. JIT2018. Rafaela, Argentina.

Orellano, S.; Rodríguez, N.; Scenna, N. J. (2019a). Optimización de playas de almacenamiento de sustancias peligrosas frente a escenarios de trench fire. CAIQ2019. Santa Fe.

Orellano, S.; Scenna, N. J. (2019b). Optimización de Layout Considerando el Diseño Basado en Riesgos. JIT2019. Rosario, Argentina.

Park K.; Shin D.; Wangyun W. (2018). Risk based 3-dimensional and multifloor plant layout optimization for liquefied natural gas (LNG) liquefaction process. Korean Journal of Chemical Engineering.

Ponzone, D. M.; Orellano, S.; Biscotti P.; Scenna N. J. (2019). Optimización de layout mediante programación matemática empleando ceibo. CAIQ2019. Santa Fe, Argentina.

Resolución 1296/2008. Condiciones mínimas que deben cumplir las plantas de elaboración y almacenamiento de biocombustibles en relación a la seguridad. Argentina

Ruiz J. P.; Grossmann I. P. (2012). A hierarchy of relaxations for nonlinear convex generalized disjunctive programming. European Journal of Operational Research 218: 3847. 
Tsai R. D.; Malstrom E. and Kuo W. (1993). Three dimensional palletization of mixed box sizes. IIE Transactions. 25: 64- 75.

Vecchietti A., Lee S., Grossmann I. E. (2003) Modeling of discrete/continuous optimization problems: characterization and formulation of disjunctions and their relaxations. Computers and Chemical Engineering 27: 433-448.

Xu G.; Papageorgiou L. G. (2007). A Construction-Based Approach to Process Plant Layout Using Mixed-Integer Optimization. Industrial \& Engineering Chemistry Research. 46: 351358.

Xu G.; Papageorgiou L. G. (2009). Process plant layout using an improvement-type algorithm. Chemical Engineering Research and Design. 87: 780-788. 